\section{Cold fusion: what's going on?}

SIR-A significant point which is not widely known, and may therefore be overlooked in neutron measurements of cold fusion rates, is the possibility of contamination by cosmic-ray-generated neutrons; these should be taken into account in the design and interpretation of experiments.

The cosmic-ray-induced neutron background arises primarily from extra-solar protons with energies above a few $\mathrm{GeV}$, which can penetrate the Earth's atmosphere and the Sun's and Earth's magnetic fields ${ }^{1}$. Primaries and secondaries reaching the surface include neutrons and other energetic particles which produce neutrons in the atmosphere and the first few metres of the surface by spallation reactions. While the spectrum contains neutrons up to energies comparable to incident particle energies, a major component is due to evaporation of neutrons from struck nuclei; at birth these have energies in the range 1-3 MeV, and appear as a knee or shoulder on an otherwise continuous energy distribution. There is also a substantial component consisting of 'thermal' neutrons, which have slowed down in the environment to a poorly equilibrated thermal distribution below energies of about $0.1 \mathrm{eV}$ as well as 'epithermal' neutrons whose distribution is roughly inversely proportional to the energy in the range $1 \mathrm{eV}$ to $1 \mathrm{MeV}$. At energies above a few $\mathrm{MeV}$, the spectrum tails off rapidly; this cascade component contains about $10 \%$ of the total. The flux of each of the low-energy components is of the order of $10^{-2}$ neutrons $\mathrm{cm}^{-2} \mathrm{~s}^{-1}$ at sea level and middle latitudes.

These figures vary according to altitude (about twice as great at 1,500 m elevation), and from time to time, mostly because of variations in atmospheric density and solar and geomagnetic field intensity. The e-folding thickness in the atmosphere is about $150 \mathrm{~g} \mathrm{~cm}^{-2}$, so that, for example, barometric pressure variations of $\pm 13 \mathrm{~mm}$ of mercury cause about $\pm 10 \%$ flux variations. Sometimes, when dealing with such a general source of neutrons, material intended as shielding, and even detector material itself, can act as a source, so that some care in this respect is called for in the measurements.

As it happens, the counting rates due to cosmic-ray-induced neutrons are of the same order of magnitude as the counting rates observed in the neutron and secondary radiation detectors in many of the measurements being made. And in detectors that disperse the spectrum, the evaporation peak in the energy distribution due to cosmic-ray-induced neutrons is at nearly the same energy as that expected from deuteron-deuteron fusion, 2.45 $\mathrm{MeV}$. These observations, coupled with the (admittedly weak. $\pm 10 \%$ ) temporal (hourly, daily) variation of the cosmic-ray-induced neutron fluxes require that this background be carefully accounted for.

Comparable neutron fluxes can be generated by accelerators, isotope sources and nuclear reactors, even at considerable distances; these contaminants of neutron measurements must also be reckoned with. Obvious means for suppressing these backgrounds are time-gating of the source, monitoring spurious sources with a second detector operated simultaneously with the detector(s) near the source under investigation, or going underground - $350 \mathrm{~g} \mathrm{~cm}^{-2}$ (two or three metres of earth or concrete on all sides) should reduce the cosmic-ray neutrons by a factor of about 10 .

Argonne National Laboratory,

9700 South Cass Avenue,

Argonne, Illinois 60439-4814, USA

1. Hayakawa, S. Cosmic Ray Physics (Wiley, New York, 1969).

Dr Carpenter, a referee of the paper by Jones et al. on page 737, provided this comment at our invitation.

The following are extracts from the substantial numbers of letters from readers offering explanations of the two series of cold fusion experiments which have been generally reported.

Editor, Nature.

SIR-From the newspaper accounts, the very small flux of neutrons generated during the experiment of Fleischmann and Pons is being taken as proof that their conclusion is not valid, and that nuclear reactions between deuterons do not occur under the conditions they describe.

But when the kinetic energy is as small as in their experiment, the neutron and proton components of the deuteron do not behave in the same way, because the nucleus of the target atom repels the proton but not the neutron. Thus, the neutron can be captured by the target nucleus while the proton, which remains outside the Coulomb barrier, will fly off.

This process, first recognized by Oppenheimer and Phillips ${ }^{1}$ in 1935 leads to a pure $(d, p)$ reaction and has a relatively high probability of occurrence, certainly much greater than that of the $(d, n)$ reaction ${ }^{2}$.

It follows that if the experiments described really brought the deuterium nuclei close enough together to interact, one should expect no neutron emission and a reaction rate much higher than that evaluated on the basis of the high-energy 1. Oppenheimer, J. R. \& Phillips, M. Phys. Rev. 48, 500
(1935).
2. Morrison, P. Experimental Physics Vol. 2 (ed. Segrè, E.)
(Wiley, New York, 1953). model.

Via O. Regnoli 10 , Rome 00152, Italy

SIR-The only recognized mechanism for nuclear fusion at ambient temperature is that induced by negative muon binding of precursor hydrogen isotope molecules as first described by Frank ${ }^{1}$ with estimates of fusion rates by $S$ akharov ${ }^{2}$ and experimental observations by $\mathrm{Alvarez}^{3}$ some 30 years ago. It is now known that more than 100 fusion events may be induced by a single muon during its lifetime of $2.2 \times 10^{-6}$ seconds in a mixture of liquid deuterium and tritium ${ }^{4}$. If this number could be increased by a factor of 1,000 , a break-even fusion reactor could be the result ${ }^{5}$.

It is tempting to interpret the recent claims in terms of this process. It should be remembered that more than 70 per cent of the cosmic-ray flux at the Earth's surface consists of positive and negative muons. There are about $200 \mathrm{~m}^{-2} \mathrm{~s}^{-1}$ with a stopping rate in an absorber of some $2 \times 10^{-5} \mathrm{~g}^{-1} \mathrm{~s}^{-1}$. The flux may be twice as great at the altitude of Salt Lake City ${ }^{6}$, which would be equivalent to more than two muons a minute in an absorbing volume of about a litre.

The rate at which fusion events could occur is limited by the rate of formation of muon-bound molecules, which is itself a sensitive function of parameters on the atomic scale, whence its dependence on resonance effects $^{7}$ and temperature ${ }^{8}$. Once a muon is captured, the resulting muonic molecule is two orders of magnitude smaller than the typical lattice spacing in solids, so that free diffusion may be expected.

The fact remains that muon-induced fusion has not yet been reported in metallic compounds of hydrogen isotopes, and indeed has been considered unlikely because of the preferential capture of muons by the heavier metal nuclei. On the hypothesis that this loss mechanism is suppressed by a resonance or band structure effect in deuterium-loaded palladium, it is possible to estimate the turnover number required to explain the effects which have been reported.

Jones et al. ${ }^{9}$ observe a neutron countrate of $4 \times 10^{-3} \mathrm{~s}^{-1}$ with a neutron detection efficiency of about $1 \%$ in a volume of 160 $\mathrm{ml}$. If the neutrons observed are products of the reaction, in a muon-deuterium molecule, of two deuterons to yield ${ }^{3} \mathrm{He}$ and a 2.45-MeV neutron, one should also allow for the equally probable reaction yielding ${ }^{3} \mathrm{H}$ and a proton, which would not have been detected by the neutron monitor. This implies five fusion events per second per litre. The required turnover number is then of the order of 100 , comparable with that already known for the case of a mixture of liquid isotopes, but significantly greater than that in pure 